\title{
Comunidade de aves no Parque Estadual da Fonte Grande, Vitória, Espírito Santo, Brasil
}

\author{
José E. Simon ${ }^{1,2}$; Saulo R. Lima ${ }^{1} \&$ Thais Cardinali ${ }^{1}$ \\ ${ }^{1}$ Laboratório de Zoologia dos Vertebrados. Faculdade Integrada de Saúde e Meio Ambiente de Vitória. Campus II, Rodovia \\ Serafim Derenze 3115. 29030-001 Vitória, Espírito Santo, Brasil. E-mail: simon@ebr.com.br \\ 2 Instituto de Pesquisas da Mata Atlântica - IPEMA. Rua Hugo Viola 1001, sala 218, 29060-420 Vitória, Espírito Santo, Brasil.
}

\begin{abstract}
Birds of the Parque Estadual da Fonte Grande, Vitória, Espírito Santo, Brazil. This paper provides the avifauna composition recorded between January and December 2003 in the Fonte Grande State Park (PEFG), municipality of Vitória, state of Espírito Santo, Brazil. We obtained a total of 120 species belonging to 37 families, after 450 hours of field work. We estimated a richness of 146 species $\left(129 \leq \mathrm{IC}_{(95 \%)} \leq 163\right)$, according to Jackknife 1 method. However, the species accumulation curve has not reached its assintotics. Four species out of 120 recorded are endemic to the Brazilian Atlantic Forest (Leucopternis Iacernulatus, Phaethornis idaliae, Thamnophilus ambiguus and Attila rufus), and one of them (L. lacernulatus) is included in the list of Brazilian birds threatened with extinction. The comparison among the three basic inventory methods (auditive, sightseeing and net capture) revealed that the sightseeing method detected the largest number of species $\left(\chi^{2}=17.55, \mathrm{p}<5 \%\right.$ ), of which 26 $(22 \%)$ were recorded exclusively by this method. This result is explained by the dominance of open landscape species in the Park, associated with the type of field work employed in this study.
\end{abstract}

KEY WORDS. Atlantic Forest; conservation; inventory.

RESUMO. O presente trabalho divulga a composição da comunidade de aves no Parque Estadual da Fonte Grande (PEFG),Vitória, Espírito Santo, com base em levantamentos de campo realizados entre janeiro e dezembro de 2003. Com um esforço de campo em torno de 450 horas, obtivemos um total de 120 espécies, distribuídas em 37 famílias. Pelo método Jackknife 1, estimou-se uma riqueza de 146 espécies $\left(129 \leq \mathrm{IC}_{(95 \%)} \leq 163\right)$, sem que, entretanto, a curva de acumulação de espécies tenha atingido a sua assíntota. Entre as espécies registradas na área de estudo, quatro são endemismos da Mata Atlântica brasileira (Leucopternis lacernulatus, Phaethornis idaliae, Thamnophilus ambiguus e Attila rufus), sendo que uma delas (L. lacernulatus) inclui-se na lista oficial da fauna brasileira ameaçada de extinção. A comparação entre os três métodos básicos utilizados no inventário das espécies (auditivo, visual e captura em redes) indicou que o método visual responsabilizou-se pela detecção de um número significativamente maior de espécies $\left(\chi^{2}=17,55 ;\right.$ g.l. $\left.=2 ; p<5 \%\right)$, com um total de 103 espécies assinaladas ( $86 \%$ do total registrado), $26(22 \%)$ das quais registradas exclusivamente por esse método. Tal resultado foi relacionado com a predominância de espécies de paisagens abertas, associada ao tempo de campo empregado por esse estudo.

PALAVRAS-CHAVE. Conservação; inventário; Mata Atlântica.

À semelhança de outras regiões do leste do Brasil, o Estado do Espírito Santo apresentava boa parte de seu território coberto por vegetação de Mata Atlântica. Em decorrência do intensificado processo de desmatamento que ocorreu no passado, o que sobrou de suas florestas nativas encontra-se hoje distribuído sob um padrão fortemente fragmentado, restando apenas 7,5\% de sua área original (FundAÇÃo SOS Mata AtLÂNTICA et al. 1998). Esta generalizada destruição deve ter provocado profundas alterações na composição da avifauna do Estado, cujos efeitos só agora começam a ser notados, seja pela extinção regional de algumas espécies, forte declínio populacional de outras ou ainda pela invasão espontânea de espécies a partir de regiões vizinhas (WILlis \& ONIKI 2002, SIMON \& LIMA 2004). Por serem, entretanto, raras as localidades inventariadas, o conhecimento de avifaunas regionais do Espírito Santo é bastante limitado, dificultando, entre outras coisas, a identificação de áreas prioritárias para a conservação da diversidade biológica.

O Espírito Santo possui muitas unidades de conservação (municipais, estaduais e federais), mas a maioria delas nunca foi devidamente estudada, ainda que inventários faunísticos (e florísticos) sejam ferramentas úteis para o adequado manejo do potencial biológico de áreas protegidas (Conservation 
International do Brasil et al. 2000, Primack \& Rodrigues 2001). As poucas unidades de conservação no Estado, para as quais levantamentos ornitológicos já foram publicados, são a Estação Biológica de Santa Lúcia (SIMON 2000, Willis \& ONIKI 2002), o Parque Estadual Paulo César Vinha (Venturini et al. 1996), a Reserva Biológica Augusto Ruschi (PARKer III \& Goerck 1997, Willis \& Oniki 2002) e a Reserva Florestal da CVRD (STOTz 1993, MARSDEN et al. 2000).

$\mathrm{O}$ presente estudo teve como objetivo inventariar a comunidade de aves no Parque Estadual da Fonte Grande, criado para proteger os últimos resquícios de Mata Atlântica da região de Vitória, ES. Sua importância como refúgio para a fauna nativa nunca foi avaliada, a não ser pelo estudo sobre Hymenoptera parasitóides (Insecta), que chamou a atenção para a ocorrência de 28 famílias, algumas das quais novas para o Brasil (Azevedo et al. 2002).

\section{MATERIAL E MÉTODOS}

\section{Área de estudo}

O Parque Estadual da Fonte Grande (PEFG), criado em agosto de 1986, localiza-se no maciço central do município de Vitória, Espírito Santo. Sua área, medindo 216 ha, estende-se por um relevo fortemente ondulado, com altitudes variando de 50 a 308 m (Secretaria Municipal do Meio Ambiente 1996, Ipema 2005).

Por sua localização, inclui-se na região fitoecológica da Floresta Ombrófila Densa (IвGE 1987), com forte influência de correntes de ar marítimas, em função da sua grande proximidade com a baía de Vitória. Sua vegetação, outrora representada por exuberantes florestas que recobriam a região de Vitória (Wied-Neuwied 1958, Saint-Hilaire 1974), caracteriza-se atualmente pelo domínio de mata secundária imersa na paisagem urbana, em sua maior parte em estágio avançado de regeneração (ca. 30 anos, Edson Valpassos e Oberdan José Pereira, comum. pess., maio/2004) (Fig. 1). Além do ambiente florestal (ca. 70\% da área total), prevalecem outras condições fitofisionômicas, como brejos, vegetação rupestre e ambientes de origem antrópica (pastagens, capoeiras e plantações abandonadas), com extensões variadas, distribuídas pela área do Parque (Secretaria Municipal do Meio Ambiente 1996).

Segundo a classificação de Köppen, o clima da região é do tipo AW, caracterizado por menor índice pluviométrico no inverno e constantes chuvas no verão. Apresenta ainda uma dinâmica particular, condicionada pelas correntes de ar Tropical Atlântico, de característica quente e úmida, e a Polar Atlântica, seca e fria, que atua principalmente no inverno (SECRETARIA Municipal do Meio Ambiente 1996).

\section{Esforço amostral}

Os trabalhos de campo foram desenvolvidos durante um período de 12 meses, entre janeiro e dezembro de 2003, através de excursões quase sempre quinzenais (22 excursões), com duração de três dias cada e participação de três membros da equipe na maioria delas. A maior parte dos trabalhos concentrou-se nas primeiras (5-10 h) e últimas (14-19 h) horas do dia, atendendo ao período de maior atividade das aves (SKIRVIN 1981, BLAKE 1992), o que totalizou cerca de 450 horas de esforço amostral para o inventário das espécies. Os trabalhos concentraram-se exclusivamente dentro dos limites do parque, aproveitando-se as trilhas existentes na área para o registro das espécies.

\section{Inventário das espécies}

Três métodos básicos foram utilizados para o inventário da comunidade de aves no PEFG: auditivo, visual e captura em redes de neblina. O método visual apoiou-se na utilização de binóculos Pentax 8 x 40 mm e Olympus 7 x 35 mm, enquanto que o método auditivo baseou-se na experiência prévia dos autores com vocalizações das espécies no Estado do Espírito Santo (SIMON 2000). Vocalizações não prontamente identificadas em campo (Elaenia spp., Myiarchus spp., etc) foram gravadas com o uso de aparelhos Sony TCM5000-EV e Marantz PDM-222, acoplado a microfone Sennheiser ME-66, para sua posterior confirmação em laboratório, através da comparação com arquivo de vozes das aves do Brasil (VielLiard 1995a, b). Essas gravações foram também empregadas durante a técnica de play-back (e.g. MARION et al. 1981), como uma ferramenta auxiliar do método auditivo, a qual limitou-se, porém, à reprodução da vocalização de uma espécie para seu contato visual pelo observador (registro visual não contabilizado pelo método visual), sendo essa técnica considerada de extrema importância na realização de inventários da avifauna em áreas de floresta tropical (Johnson et al. 1981, Parker III 1991). Por sua vez, o método de captura em redes incluiu de cinco a dez redes de neblina (dimensões de $12 \mathrm{x}$ $2,5 \mathrm{~m}$, com malha de $36 \mathrm{~mm}$ ) empregadas em todas as excursões, permanecendo abertas durante todo o dia (6-18 horas), o que totalizou um esforço de captura de $198.720 \mathrm{~m}^{2} . \mathrm{h}$ (sensu Straube \& Bianconi 2002). As redes foram sempre montadas próximo ao solo e em posições variadas na área de estudo, atendendo igualmente as áreas antrópicas e o ambiente florestal. A freqüência de captura em rede das espécies foi calculada pela razão entre o número de meses em que cada uma delas foi capturada e o número total de meses trabalhados.

Com base nos dados levantados, comparou-se os três métodos básicos utilizados em nosso inventário (auditivo, visual e captura em redes), visando avaliar qual deles responsabilizou-se pela detecção do maior número de espécies no período estudado.

Quando necessário, o conhecimento de caracteres diagnósticos para a identificação de espécies crípticas apoiou-se em bibliografias especializadas (Meyer de Schauensee 1970, Grantsau 1988, Ridgely \& Turdor 1989, 1994, Sick 1997, Souza 2002) e em consultas à coleção ornitológica do Museu de Biologia Prof. Mello Leitão (MBML) e Museu Nacional do Rio de Janeiro (MNRJ). Eventuais coletas foram realizadas com redes de neblina para dirimir dúvidas taxonômicas, sendo que todo material coletado encontra-se depositado no MBML (Santa Teresa, Espírito Santo). A seqüência sistemática e a nomenclatura científica-po- 


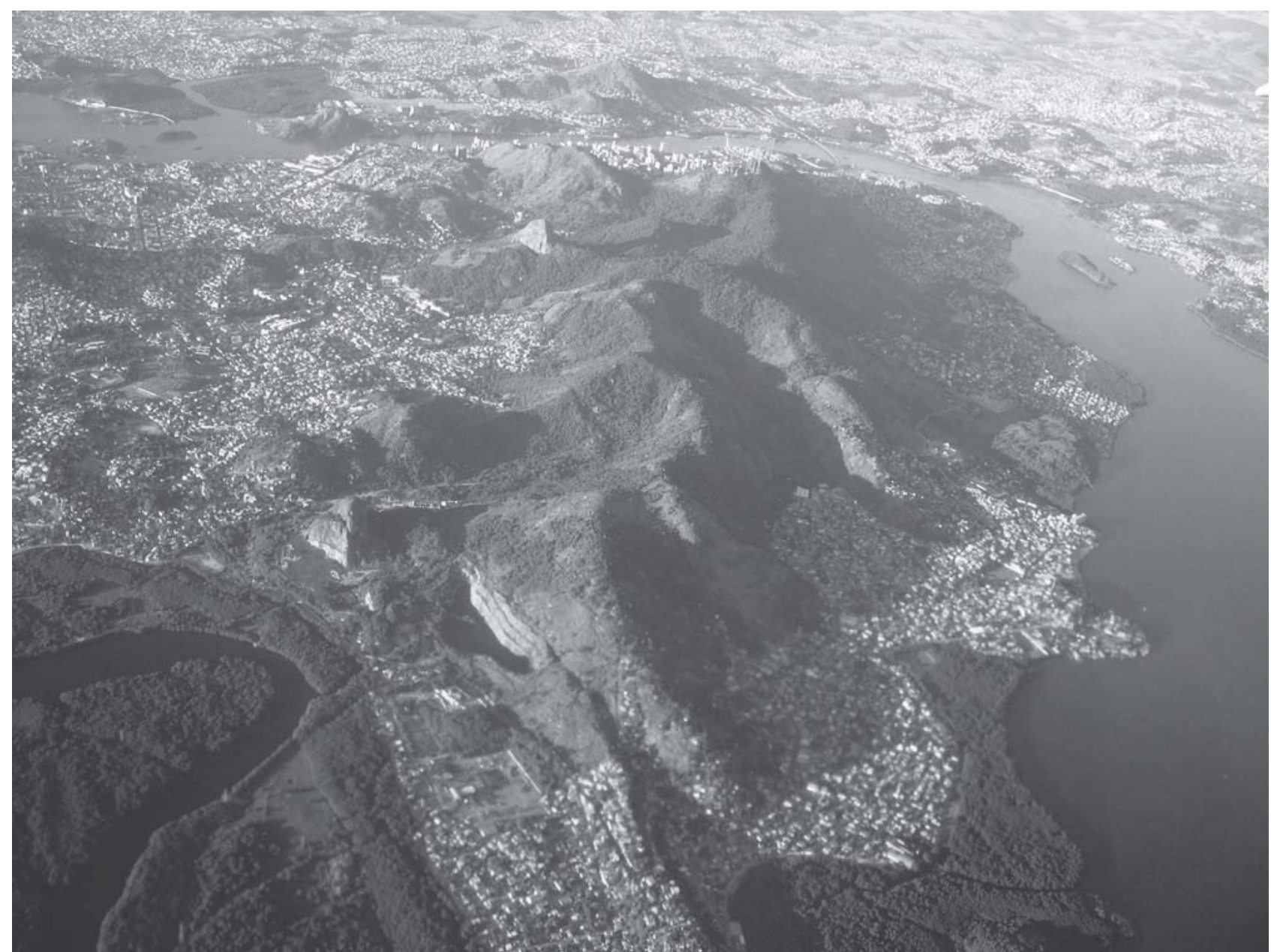

Figura 1. Vista geral do Parque Estadual da Fonte Grande, situado no maciço central do município de Vitória, Espírito Santo. Foto aérea: J.E. Simon.

pular apresentadas na tabela I seguem a Lista das Aves do Brasil, organizada pelo Comitê Brasileiro de Registros Ornitológicos (2005). A citação de Numida meleagris nessa tabela segue Sick (1997). Adotou-se PARKER III et al. (1996) combinado com SicK (1997) para a citação dos endemismos da Mata Atlântica brasileira. Por fim, a referência a espécies ameaçadas de extinção seguiu a Lista Oficial de Espécies da Fauna Brasileira Ameaçada de Extinção (Iвama 2003).

\section{Análise dos dados}

A frequiência de ocorrência (FO) de cada espécie na área de estudo foi determinada com base na seguinte equação (D'Angelo Neto et al. 1998): FO = N x 100/NT; onde N = número de meses que a espécie foi registrada; $\mathrm{NT}$ = número total de meses trabalhados $(\mathrm{NT}=12)$. Logo, a FO relaciona a proporção dos meses em que a espécie foi detectada com o número total de meses empregados no trabalho, indicando a probabilidade de uma espécies ser encontrada na área de estudo. Com o cál- culo de FO, dado em porcentagem, foi possível categorizar as espécies em: I) pouco comum (8,3 a 33,3\%): espécie registrada entre um e quatro meses; II) comum (41,6 a 66,6\%): espécie registrada entre cinco e oito meses; e III) muito comum (75 a 100\%): espécie registrada entre nove e doze meses.

A estimativa da riqueza de espécies calculada para a área e a respectiva curva de acumulação de espécies foram obtidas pelo método Jackknife 1 (Smith \& Belle 1984, Krebs 1999) com auxílio dos softwares Excel 5.0, EstimateS 6.01b e Statistica 6.0 (Colwell 1997). Os cálculos foram realizados com base em 50 randomizações (runs), considerando uma amostra como o esforço de campo mensal (seis dias) aplicado para o levantamento das espécies (total de 12 amostras). O intervalo de confiança da riqueza estimada foi determinado pela seguinte equação (Zar 1999): IC (95\%) = X \pm t (a, gl) Sx; onde: IC (95\%): intervalo de confiança da riqueza estimada pelo método Jackknife 1 ; X: riqueza estimada pelo método Jackknife $1 ; \mathrm{t}_{(\alpha, \mathrm{gl})}$ : valor de $\mathrm{t}$ da

Revista Brasileira de Zoologia 24 (1): 121-132, março 2007 
distribuição de Student ( $\alpha=5 \%$ para o teste bicaudal); Sx: erro padrão da riqueza estimada.

O teste Qui-quadrado $\left(\chi^{2}\right)$ foi empregado para a comparação entre o número de espécies detectadas pelos três métodos básicos de inventário da avifauna do PEFG.

\section{RESULTADOS}

Com base nos trabalhos de campo, um total de 120 espécies de aves, distribuídas em 37 famílias, foram registradas na área de estudo (Tab. I). Desse total, 55 (46\%) espécies pertencem ao grupo dos não-passeriformes e 65 (54\%), à ordem Passeriformes. Entre as famílias de aves não-passeriformes, Falconidae e Columbidae destacam-se como as duas famílias mais representadas, com sete espécies cada. Por sua vez, as famílias Tyrannidae e Thraupidae, com 21 e oito espécies, respectivamente, destacam-se como as duas mais representadas da ordem Passeriformes. A maior parte das espécies (75\%) foi registrada nos seis primeiros meses, porém, o número de espécies a cada nova excursão aumentou até o encerramento dos trabalhos de campo. O método Jackknife 1 estimou uma riqueza de 146 espécies, com intervalo de confiança entre 129 e 163, sem que, entretanto, a curva de acumulação de espécies tenha atingido a sua assíntota (Fig. 2)

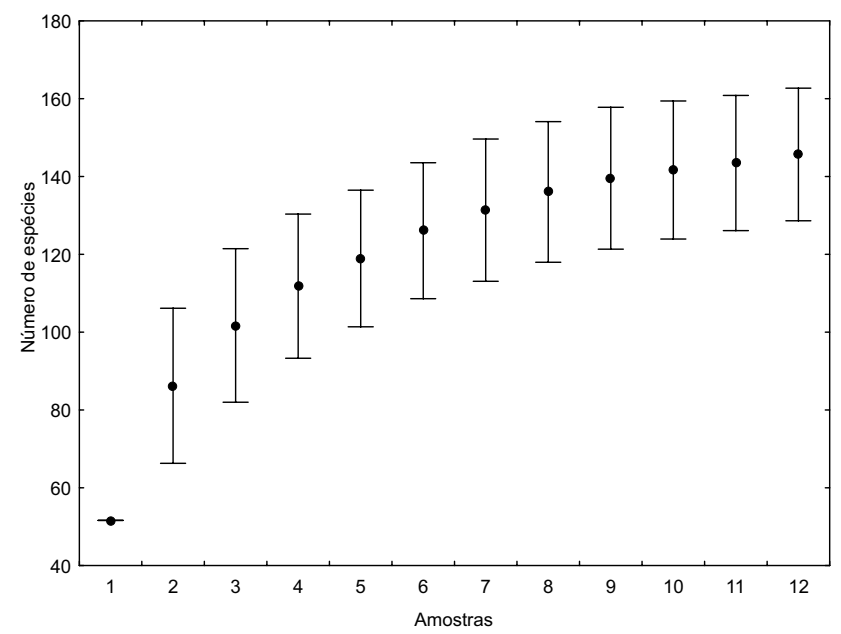

Figura 2. Curva de acumulação da riqueza estimada das espécies de aves para o Parque Estadual da Fonte Grande, Vitória, Espírito Santo (estimador Jackknife 1). Linhas verticais representam o intervalo de confiança (95\%) em torno da média estimada.

Das 120 espécies registradas, 41 (34\%) possuem freqüência de ocorrência muito comum na área, sendo que das 79 restantes, 18 (15\%) são comuns e 61 (51\%), pouco comuns (Fig. 3). Entre as espécies de ocorrência muito comum, sobressaem tanto as não-florestais (e.g. Milvago chimachima, Nyctidromus albicollis e Pitangus sulphuratus) como as florestais (e.g. Myrmotherula

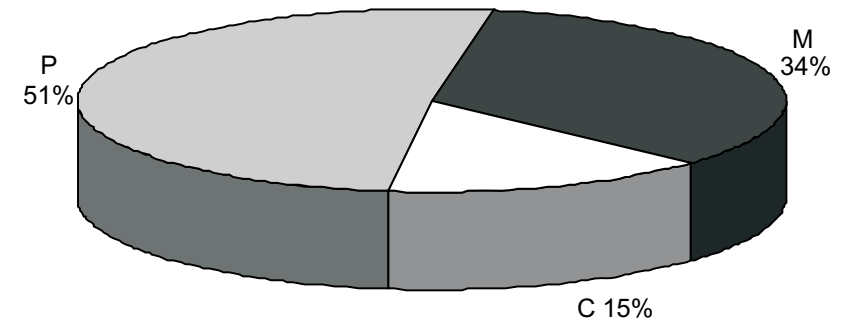

Figura 3. Distribuição da freqüência de ocorrência da avifauna no Parque Estadual da Fonte Grande, Vitória, Espírito Santo. (P) Pouco comum, (C) comum, (M) muito comum.

axillaris, Tolmomyias flaviventris e Parula pitiayumi). Por outro lado, espécies como Crypturellus tataupa (Tinamidae), Leucopternis lacernulatus (Accipitridae), Primolius maracana (Psittacidae), Nyctibius griseus (Nyctibiidae), Attila rufus (Tyrannidae), Platycichla flavipes (Turdidae), Turdus rufiventris (Turdidae) e Sicalis flaveola (Emberizidae) revelaram-se pouco comuns na área (Tab. I).

A comparação entre os três métodos básicos utilizados no inventário das espécies (auditivo, visual e captura em redes) indicou que o método visual responsabilizou-se pela detecção de um número significativamente maior de espécies $\left(\chi^{2}=17,55\right.$; g.l. $=2 ; \mathrm{p}<5 \%$ ), com um total de 103 espécies assinaladas (86\% do total registrado), 26 (22\%) das quais registradas exclusivamente por esse método (Fig. 4). O segundo lugar coube ao método auditivo, com um total de 77 espécies registradas (64\%), oito (7\%) delas exclusivas desse método. Um total de 51 (42\%) espécies foi detectado pelo método de rede, com sete $(6 \%)$ espécies dele exclusivas (Fig. 4).

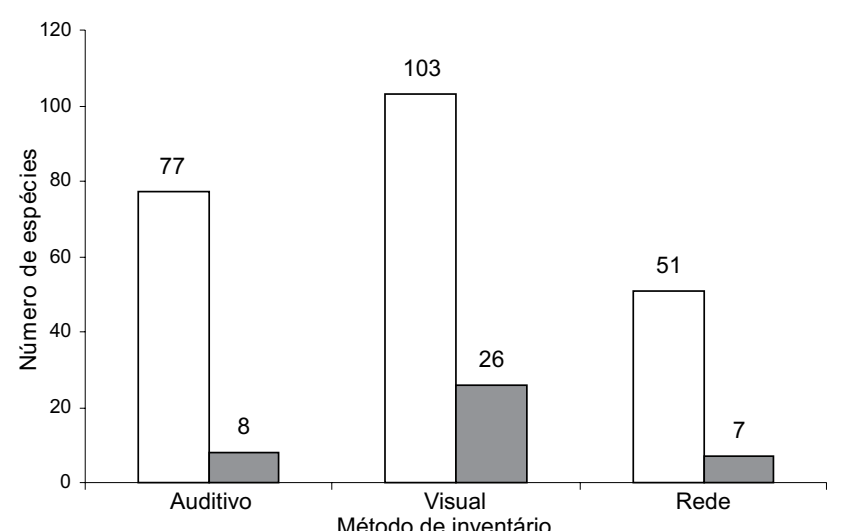

Figura 4. Número de espécies de aves detectadas pelos três diferentes métodos de inventário utilizados nesse estudo. Coluna clara: total de espécies, coluna escura: espécies exclusivas.

Entre as 51 espécies registradas pelo método de rede, Elaenia flavogaster foi a mais comum, com uma freqüência de captura na área de estudo igual a 0,75. Em seguida, aparecem Tolmomyias 
Tabela I. Composição da avifauna no Parque Estadual da Fonte Grande, município de Vitória, Espírito Santo.

\begin{tabular}{|c|c|c|c|c|}
\hline Família/espécie & Nome popular & FO & MI & FC \\
\hline \multicolumn{5}{|l|}{ Não-passeriformes } \\
\hline \multicolumn{5}{|l|}{ Tinamidae } \\
\hline Crypturellus tataupa (Temminck, 1815) & inhambu-chintã & $P$ & $a v$ & \\
\hline \multicolumn{5}{|l|}{ Cracidae } \\
\hline Ortalis guttata (Spix, 1825) & aracuã & $P$ & a & \\
\hline \multicolumn{5}{|l|}{ Numididae } \\
\hline Numida meleagris (Linnaeus, 1758) * & galinha-d' angola & $\mathrm{C}$ & $a v$ & \\
\hline \multicolumn{5}{|l|}{ Ardeidae } \\
\hline Tigrisoma lineatum (Boddaert, 1783) & socó-boi & $P$ & $a v$ & \\
\hline \multicolumn{5}{|l|}{ Cathartidae } \\
\hline Cathartes aura (Linnaeus, 1758) & urubu-de-cabeça-vermelha & M & $v$ & \\
\hline Coragyps atratus (Bechstein, 1793) & urubu-de-cabeça-preta & M & $v$ & \\
\hline \multicolumn{5}{|l|}{ Accipitridae } \\
\hline Geranospiza caerulescens (Vieillot, 1817) & gavião-pernilongo & $P$ & $v$ & \\
\hline Leucopternis lacernulatus (Temminck, 1827) En, VU & gavião-pombo-pequeno & $P$ & v & \\
\hline Rupornis magnirostris (Gmelin, 1788) & gavião-carijó & M & $a v$ & \\
\hline Buteo albicaudatus Vieillot, 1816 & gavião-de-rabo-branco & $\mathrm{C}$ & v & \\
\hline Buteo brachyurus Vieillot, 1816 & gavião-de-cauda-curta & M & $v$ & \\
\hline Buteo albonotatus Kaup, 1847 & gavião-de-rabo-barrado & $P$ & $\mathrm{v}$ & \\
\hline \multicolumn{5}{|l|}{ Falconidae } \\
\hline Caracara plancus (Miller, 1777) & caracará & M & $a v$ & \\
\hline Milvago chimachima (Vieillot, 1816) & carrapateiro & M & $a v$ & \\
\hline Herpetotheres cachinnans (Linnaeus, 1758) & acauã & M & $a v$ & \\
\hline Falco sparverius Linnaeus, 1758 & quiriquiri & $P$ & $a v$ & \\
\hline Falco rufigularis Daudin, 1800 & cauré & $P$ & $v$ & \\
\hline Falco femoralis Temminck, 1822 & falcão-de-coleira & $P$ & v & \\
\hline Falco peregrinus Tunstall, 1771 & falcão-peregrino & $P$ & v & \\
\hline \multicolumn{5}{|l|}{ Rallidae } \\
\hline Aramides cajanea (Statius Muller, 1776) & saracura-três-potes & $\mathrm{M}$ & $a v$ & \\
\hline \multicolumn{5}{|l|}{ Columbidae } \\
\hline Columbina talpacoti (Temminck, 1811) & rolinha-roxa & M & $a v r$ & 0,42 \\
\hline Columbina squammata (Lesson, 1831) & fogo-apagou & M & $a v$ & \\
\hline Columbina picui (Temminck, 1813) & rolinha-picui & $P$ & $v$ & \\
\hline Claravis pretiosa (Ferrari-Perez, 1886) & pararu-azul & $P$ & $\mathrm{r}$ & 0,08 \\
\hline Columba livia Gmelin, 1789 & pombo-doméstico & $P$ & $\mathrm{v}$ & \\
\hline Patagioenas picazuro (Temminck, 1813) & pombão & $P$ & $a v$ & \\
\hline Leptotila verreauxi Bonaparte, 1855 & juriti-pupu & $\mathrm{M}$ & $a \vee r$ & 0,17 \\
\hline \multicolumn{5}{|l|}{ Psittacidae } \\
\hline Ara ararauna (Linnaeus, 1758) * & arara-canindé & $\mathrm{C}$ & $a v$ & \\
\hline Primolius maracana (Vieillot, 1816) & maracanã-do-buriti & $P$ & $a v$ & \\
\hline Aratinga leucophthalma (Statius Muller, 1776) & periquitão-maracanã & $P$ & $a v$ & \\
\hline Brotogeris tirica (Gmelin, 1788) En & periquito-rico & $P$ & $a v$ & \\
\hline Pionus maximiliani (Kuhl, 1820) & maitaca-verde & M & $a v$ & \\
\hline
\end{tabular}


Tabela I. Continuação.

\begin{tabular}{|c|c|c|c|c|}
\hline Família/espécie & Nome popular & $\mathrm{FO}$ & $\mathrm{Ml}$ & FC \\
\hline \multicolumn{5}{|l|}{ Cuculidae } \\
\hline Piaya cayana (Linnaeus, 1766) & alma-de-gato & $\mathrm{P}$ & $a v$ & \\
\hline Crotophaga ani Linnaeus, 1758 & anu-preto & M & $a v r$ & 0,17 \\
\hline Guira guira (Gmelin, 1788) & anu-branco & $\mathrm{P}$ & $v$ & \\
\hline \multicolumn{5}{|l|}{ Tytonidae } \\
\hline Tyto alba (Scopoli, 1769) & coruja-da-igreja & $\mathrm{C}$ & $a v$ & \\
\hline \multicolumn{5}{|l|}{ Strigidae } \\
\hline Megascops choliba (Vieillot, 1817) & corujinha-do-mato & M & $\mathrm{a} v$ & \\
\hline Pulsatrix cf. perspicillata (Latham, 1790) & murucututu & $\mathrm{P}$ & a & \\
\hline Glaucidium brasilianum (Gmelin, 1788) & caburé & $\mathrm{P}$ & $v$ & \\
\hline Athene cunicularia (Molina, 1782) & coruja-buraqueira & $\mathrm{P}$ & $a v$ & \\
\hline Rhinoptynx clamator (Vieillot, 1808) & coruja-orelhuda & $\mathrm{P}$ & $v$ & \\
\hline \multicolumn{5}{|l|}{ Nyctibiidae } \\
\hline Nyctibius griseus (Gmelin, 1789) & mãe-da-lua & $\mathrm{P}$ & $v$ & \\
\hline \multicolumn{5}{|l|}{ Caprimulgidae } \\
\hline Nyctidromus albicollis (Gmelin, 1789) & bacurau & M & $a v r$ & 0,25 \\
\hline Hydropsalis torquata (Gmelin, 1789) & bacurau-tesoura & M & $a v$ & \\
\hline \multicolumn{5}{|l|}{ Apodidae } \\
\hline Streptoprocne zonaris (Shaw, 1796) & taperuçu-de-coleira-branca & $\mathrm{P}$ & $v$ & \\
\hline \multicolumn{5}{|l|}{ Trochilidae } \\
\hline Glaucis hirsutus (Gmelin, 1788) & balança-rabo-de-bico-torto & $\mathrm{P}$ & $v$ & \\
\hline Phaethornis idaliae (Bourcier \& Mulsant, 1856) En & rabo-branco-mirim & M & $v r$ & 0,08 \\
\hline Eupetomena macroura (Gmelin, 1788) & beija-flor-tesoura & M & $v r$ & 0,42 \\
\hline Chlorestes notata (Reich, 1793) & beija-flor-de-garganta-azul & $\mathrm{P}$ & $r$ & 0,08 \\
\hline Chlorostilbon aureoventris (d'Orbigny \& Lafresnaye, 1838) & besourinho-de-bico-vemelho & $P$ & $\mathrm{vr}$ & 0,08 \\
\hline Amazilia fimbriata (Gmelin, 1788) & beija-flor-de-garganta-verde & M & $v r$ & 0,42 \\
\hline \multicolumn{5}{|l|}{ Alcedinidae } \\
\hline Ceryle torquatus (Linnaeus, 1766) & martim-pescador-grande & $\mathrm{P}$ & $v$ & \\
\hline \multicolumn{5}{|l|}{ Picidae } \\
\hline Picumnus cirratus Temminck, 1825 & pica-pau-anão-barrado & M & $a \vee r$ & 0,42 \\
\hline Colaptes campestris (Vieillot, 1818) & pica-pau-do-campo & C & $a v$ & \\
\hline Celeus flavescens (Gmelin, 1788) & pica-pau-de-cabeça-amarela & $\mathrm{C}$ & $a \vee r$ & 0,08 \\
\hline \multicolumn{5}{|l|}{ Passeriformes } \\
\hline \multicolumn{5}{|l|}{ Thamnophilidae } \\
\hline Thamnophilus ambiguus Swainson, 1825 & choca-de-sooretama & M & $a \vee r$ & 0,08 \\
\hline Myrmotherula axillaris (Vieillot, 1817) & choquinha-de-flanco-branco & M & $a \vee r$ & 0,33 \\
\hline Formicivora sp. & papa-formiga & $\mathrm{P}$ & a & \\
\hline \multicolumn{5}{|l|}{ Dendrocolaptidae } \\
\hline Xiphorhynchus picus (Gmelin, 1788) & arapaçu-de-bico-branco & $P$ & $\mathrm{v}$ & \\
\hline \multicolumn{5}{|l|}{ Furnariidae } \\
\hline Synallaxis frontalis Pelzeln, 1859 & petrim & $\mathrm{C}$ & $a \vee r$ & 0,08 \\
\hline Phacellodomus rufifrons (Wied, 1821) & joão-de-pau & $P$ & $a v$ & \\
\hline Xenops rutilans Temminck, 1821 & bico-virado-carijó & $P$ & $r$ & 0,08 \\
\hline & & & & Continu \\
\hline
\end{tabular}


Tabela I. Continuação.

Família/espécie

Tyrannidae

Leptopogon amaurocephalus Tschudi, 1846

Todirostrum cinereum (Linnaeus, 1766)

Phyllomyias fasciatus (Thunberg, 1822)

Elaenia flavogaster (Thunberg, 1822)

Elaenia albiceps (d'Orbigny \& Lafresnaye, 1837)

Elaenia mesoleuca (Deppe, 1830)

Ornithion inerme Hartlaub, 1853

Camptostoma obsoletum (Temminck, 1824)

Tolmomyias flaviventris (Wied, 1831)

Myiophobus fasciatus (Statius Muller, 1776)

Cnemotriccus fuscatus (Wied, 1831)

Satrapa icterophrys (Vieillot, 1818)

Myiozetetes similis (Spix, 1825)

Pitangus sulphuratus (Linnaeus, 1766)

Myiodynastes maculatus (Statius Muller, 1776)

Megarynchus pitangua (Linnaeus, 1766)

Tyrannus melancholicus Vieillot, 1819

Sirystes sibilator (Vieillot, 1818)

Myiarchus ferox (Gmelin, 1789)

Myiarchus tyrannulus (Statius Muller, 1776)

Attila rufus (Vieillot, 1819) En

Tityridae

Pachyramphus polychopterus (Vieillot, 1818)

Pachyramphus marginatus (Lichtenstein, 1823)

Pachyramphus validus (Lichtenstein, 1823)

Vireonidae

Vireo olivaceus (Linnaeus, 1766)

Hirundinidae

Progne chalybea (Gmelin, 1789)

Pygochelidon cyanoleuca (Vieillot, 1817)

Troglodytidae

Thryothorus genibarbis Swainson, 1838

Troglodytes musculus Naumann, 1823

Turdidae

Platycichla flavipes (Vieillot, 1818)

Turdus rufiventris Vieillot, 1818

Turdus leucomelas Vieillot, 1818

Turdus amaurochalinus Cabanis, 1850

Mimidae

Mimus saturninus (Lichtenstein, 1823)

Coerebidae

Coereba flaveola (Linnaeus, 1758)

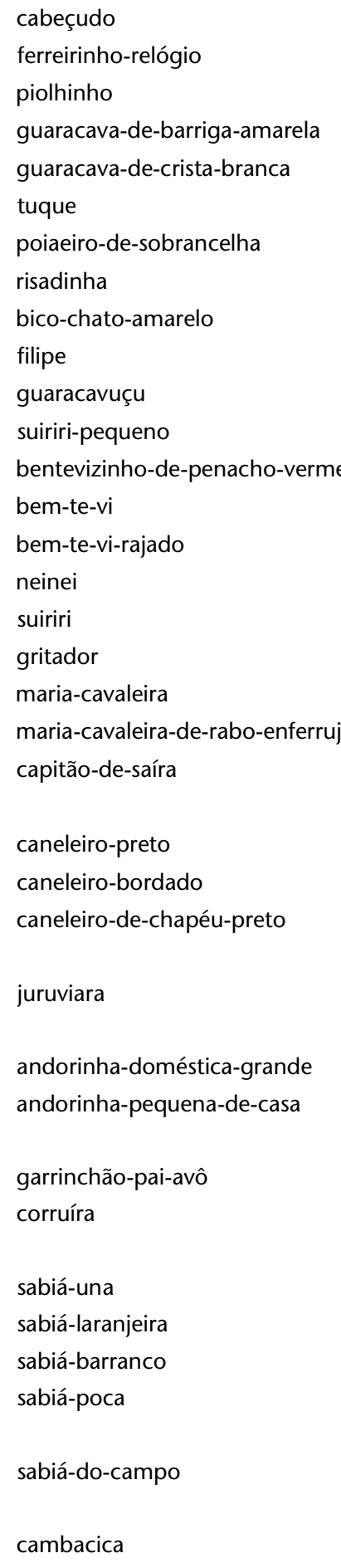

$\begin{array}{llll}\text { Nome popular } & \text { FO } & \text { MI } & \text { FC }\end{array}$

\begin{tabular}{|c|c|c|}
\hline$P$ & $a v r$ & 0,08 \\
\hline M & $a v r$ & 0,17 \\
\hline $\mathrm{P}$ & $\mathrm{a}$ & \\
\hline M & $a \vee r$ & 0,75 \\
\hline$C$ & $r$ & 0,25 \\
\hline$P$ & $r$ & 0,25 \\
\hline$C$ & $r$ & 0,25 \\
\hline M & $a v r$ & 0,25 \\
\hline M & $a \vee r$ & 0,58 \\
\hline$P$ & $a r$ & 0,08 \\
\hline$P$ & $a \vee r$ & 0,17 \\
\hline$P$ & $r$ & 0,08 \\
\hline M & $a v r$ & 0,08 \\
\hline M & $a \vee r$ & 0,42 \\
\hline$P$ & $a v$ & \\
\hline M & $a v$ & \\
\hline M & $a \vee r$ & 0,42 \\
\hline$P$ & $a$ & \\
\hline$C$ & $a \vee r$ & 0,17 \\
\hline$P$ & $v r$ & 0,17 \\
\hline $\mathrm{P}$ & $a \vee r$ & 0,08 \\
\hline$C$ & $a$ & \\
\hline$C$ & $a \vee r$ & 0,17 \\
\hline $\mathrm{P}$ & v & \\
\hline M & $a \vee r$ & 0,25 \\
\hline$P$ & $a v$ & \\
\hline$C$ & $a \vee r$ & 0,08 \\
\hline $\mathrm{P}$ & $a$ & \\
\hline M & $a \vee r$ & 0,50 \\
\hline $\mathrm{P}$ & v & \\
\hline $\mathrm{P}$ & v & \\
\hline $\mathrm{P}$ & $a \vee r$ & 0,08 \\
\hline$C$ & $a v r$ & 0,25 \\
\hline$P$ & v & \\
\hline \multirow[t]{2}{*}{$M$} & $a v r$ & 0,5 \\
\hline & & Continua \\
\hline
\end{tabular}


Tabela I. Continuação.

\begin{tabular}{|c|c|c|c|c|}
\hline Família/espécie & Nome popular & FO & MI & FC \\
\hline \multicolumn{5}{|l|}{ Thraupidae } \\
\hline Nemosia pileata (Boddaert, 1783) & saíra-de-chapéu-preto & $\mathrm{C}$ & $v r$ & 0,17 \\
\hline Tachyphonus coronatus (Vieillot, 1822) En & tiê-preto & $P$ & $v r$ & 0,17 \\
\hline Thraupis sayaca (Linnaeus, 1766) & sanhaçu-cinzento & $P$ & $a \vee r$ & 0,17 \\
\hline Thraupis palmarum (Wied, 1823) & sanhaçu-do-coqueiro & $P$ & $a v$ & \\
\hline Tersina viridis (Illiger, 1811) & saí-andorinha & $P$ & $v$ & \\
\hline Dacnis cayana (Linnaeus, 1766) & saí-azul & $\mathrm{M}$ & $v r$ & 0,33 \\
\hline Cyanerpes cyaneus (Linnaeus, 1766) & saíra-beija-flor & $P$ & $v r$ & 0,08 \\
\hline Conirostrum speciosum (Temminck, 1824) & figuinha-de-rabo-castanho & $\mathrm{M}$ & $v r$ & 0,08 \\
\hline \multicolumn{5}{|l|}{ Emberizidae } \\
\hline Zonotrichia capensis (Statius Muller, 1776) & tico-tico & $\mathrm{P}$ & $a \vee r$ & 0,08 \\
\hline Ammodramus humeralis (Bosc, 1792) & tico-tico-do-campo & $P$ & a & \\
\hline Sicalis flaveola (Linnaeus, 1766) & canário-da-terra-verdadeiro & $P$ & $a v$ & \\
\hline Volatinia jacarina (Linnaeus, 1766) & tiziu & M & $a \vee r$ & 0,17 \\
\hline Sporophila caerulescens (Vieillot, 1823) & coleirinho & $\mathrm{C}$ & $a v$ & \\
\hline Coryphospingus pileatus (Wied, 1821) & tico-tico-rei-cinza & M & $a \vee r$ & 0,33 \\
\hline \multicolumn{5}{|l|}{ Cardinalidae } \\
\hline Saltator maximus (Statius Muller, 1776) & tempera-viola & $\mathrm{C}$ & $a \vee r$ & 0,08 \\
\hline Saltator similis d'Orbigny \& Lafresnaye, 1837 * & trinca-ferro-verdadeiro & $P$ & $\mathrm{a} v$ & \\
\hline \multicolumn{5}{|l|}{ Parulidae } \\
\hline Parula pitiayumi (Vieillot, 1817) & mariquita & M & $a v$ & \\
\hline Geothlypis aequinoctialis (Gmelin, 1789) & pia-cobra & $\mathrm{P}$ & $v$ & \\
\hline \multicolumn{5}{|l|}{ Icteridae } \\
\hline Psarocolius decumanus (Pallas, 1769) & japu & $\mathrm{P}$ & $a v$ & \\
\hline \multicolumn{5}{|l|}{ Fringillidae } \\
\hline Euphonia chlorotica (Linnaeus, 1766) & fim-fim & M & $a v$ & \\
\hline Euphonia violacea (Linnaeus, 1758) & gaturamo-verdadeiro & M & $a \vee r$ & 0,42 \\
\hline Euphonia cyanocephala & gaturamo-rei & $\mathrm{P}$ & $a v$ & \\
\hline \multicolumn{5}{|l|}{ Estrildidae } \\
\hline Estrilda astrild (Linnaeus, 1758) & bico-de-lacre & $\mathrm{M}$ & $a v$ & \\
\hline
\end{tabular}

A seqüência sistemática e a nomenclatura científica-popular seguem a Lista das Aves do Brasil (CBRO 2005). A citação de Numida meleagris segue Sick (1997). Nomes científicos seguidos de "En" correspondem a endemismos da Mata Atlântica brasileira (segundo PARKER III et al. 1996 combinado com SICK 1997); aqueles seguidos por asterisco $\left({ }^{*}\right)$ correspondem a táxon alóctone para a área do parque (procedência de cativeiro). A referência ao status de conservação de Leucopternis lacernulatus segue a Lista Oficial de Espécies da Fauna Brasileira Ameaçada de Extinção (IBAMA 2003). FO (Freqüência de Ocorrência): (P) pouco comum, (C) comum, (M) muito comum (vide metodologia para detalhes). MI (Método de Inventário): (a) auditivo, (v) visual, (r) rede. FC (Frequência de Captura em Rede): razão entre o número de meses que a espécie foi capturada e o número total de meses do período de estudo.

flaviventris e Troglodytes musculus, com freqüência de 0,58 e 0,50, respectivamente. Entre as espécies com baixa freqüência de captura $(0,08)$, destacam-se, por exemplo, Celeus flavescens, Leptopogon amaurocephalus, Attila rufus e Zonotrichia capensis (Tab. I).

Em adição, do total das espécies registradas para o parque, quatro são consideradas endemismos da Mata Atlântica brasileira: Leucopternis lacernulatus, Phaethornis idaliae, Thamnophilus ambiguus e Attila rufus. Leucopternis lacernulatus ainda inclui-se na Lista Oficial de Espécies da Fauna Brasileira
Ameaçada de Extinção, registrada na área com base no contato visual de um único indivíduo, pousado em uma embaúba (Cecropia sp.) na borda da mata, em 18/VI/2003.

\section{DISCUSSÃO}

\section{Comunidade de aves no Parque}

O presente estudo aperfeiçoa substancialmente o conhecimento sobre a composição da avifauna do PEFG, somando 98 espécies ao levantamento prévio disponível para a área (SE- 
cretaria Municipal do Meio Ambiente 1996). Das 31 espécies anteriormente relacionadas para o Parque (quatro das quais não determinadas ao nível específico), cinco (Penelope superciliaris, Furnarius rufus, Sporophila lineola, Gnorimopsar chopi e Passer domesticus) não foram detectadas em campo pelo presente estudo, em função, provavelmente, de sua ocorrência ocasional na área (e.g. Sporophila lineola), seu registro fora dos limites do parque (e.g. Furnarius rufus) (considerando que o estudo mencionado envolveu áreas do entorno) ou, ainda, por possível extinção local (Penelope superciliaris).

A ausência de dados históricos limita a compreensão sobre a composição original da avifauna do PEFG, dificultando reconhecer os elementos alóctones entre as espécies registradas, seja pelo processo espontâneo de colonização de novas áreas (expansão geográfica) ou pela procedência de cativeiro (soltura ou fuga de espécimes). Contudo, cinco delas (Numida meleagris, Columba livia, Ara ararauna, Saltator similis e Estrida astrild) correspondem a táxons alienígenas, sendo que Numida meleagris, Ara ararauna e Saltator similis procedem de cativeiro, conforme entrevistas com guardas-parque e moradores da região.

Embora representando $18,5 \%$ do total da avifauna conhecida para o Espírito Santo (SIMON et al. 2005), o conjunto das 120 espécies assinaladas pelo presente estudo não deve corresponder à composição da comunidade original da área, considerando que no passado ela deveria ser dominada por elementos florestais, em função das pujantes florestas que cobriam a região de Vitória (Wied 1958, SAint-Hilaire 1974). De fato, poucas foram as espécies estritamente florestais registradas por esse estudo (e.g. Myrmotherula axillaris, Attila rufus, Leptopogon amaurocephalus, Leucopternis lacernulatus, Celeus flavescens), as quais, em sua maioria, apresentaram baixa freqüência de ocorrência no PEFG. Esse processo tem sido interpretado como efeito de uma dinâmica particular de substituição de avifaunas regionais, em decorrência da modificação de ambientes naturais (Straube \& Urben-Filho 2001).

Sendo assim, várias podem ter sido as espécies florestais que se extinguiram na área do parque, como Ramphastos vitellinus, Trogon viridis, Carpornis melanocephalus e Pipra rubrocapilla, uma vez que as mesmas ocorrem na Reserva Biológica de Duas Bocas (2.920 ha) (Collar et al. 1992), situada a $15 \mathrm{~km}$ do PEFG. Logo, o parque não deve ter atuado como um refúgio para essas espécies, dada a acentuada deterioração e fragmentação florestal que a área sofreu no passado.

Considerando que a curva de riqueza estimada de espécies não atingiu a sua assíntota, é esperado que novas espécies venham a ser registradas no PEFG. De fato, sabe-se que áreas perturbadas sofrem um ganho constante de espécies (WiLlis \& ONIKI 2002) e que curvas de riqueza crescem conforme aumenta o esforço amostral na área de estudo (SANTOS 2003).

\section{O papel do PEFG na conservação da avifauna local}

Com base na comunidade de aves registrada em nosso estudo, presume-se que o parque tenha, de fato, sofrido uma expressiva perda de espécies, figurando-se atualmente como uma área de "floresta vazia" (REDFord 1992), pelo menos para o grupo faunístico estudado. De modo geral, grandes frugívoros (cracídeos, psitacídeos, trogonídeos, ranfastídeos) e pequenos insetívoros do solo e do subbosque (formicarídeos, taminofilídeos, dendrocolaptídeos, furnarídeos) compõem o perfil das espécies mais afetadas com a fragmentação e a estrutura secundária da vegetação da Mata Atlântica (Willis 1979, Aleixo \& Vielliard 1995, Christiansen \& Pitter 1997, Aleixo 2001, Ribon et al. 2003), corroborando os resultados do presente estudo, em vista da notória ausência ou raridade dessas guildas no PEFG.

A perda de espécies em florestas isoladas, em função da fragmentação dos ecossistemas pela ação do homem, é decorrente de inúmeras causas, entre elas, a redução do hábitat, eliminação de microhábitats, estreita valência ecológica das espécies e fatores associados ao efeito de borda, os quais provocam drásticas mudanças físicas e biológicas no ecossistema (BIERREGAARD et al. 1992, MuRCIA 1995). Por tratar-se de uma floresta urbana, fatores como, caça, incêndio e presença de animais domésticos (gado, cachorros e gatos), são comuns dentro dos limites do PEFG, comprometendo ainda mais a persistência da fauna nativa. Com efeito, a criação de unidades de conservação, por si só, não garante a sustentabilidade da biodiversidade local, exigindo, entre outras coisas, o desenvolvimento de programas de educação ambiental junto aos moradores do entorno e maiores investimentos na fiscalização da área (ChIARELlo 2000).

\section{Comparação entre os métodos básicos de inventário}

Em florestas tropicais, onde a vegetação costuma oferecer baixa visibilidade para um observador, o levantamento de aves pelo método auditivo é considerado extremamente importante (Develey 2003), chegando a se responsabilizar por até 90\% das espécies registradas em um inventário (SiCK 1997). Inclusive é altamente recomendado para amostragens quantitativas (point counts), pelo menor esforço que demanda na detecção das espécies (Aleixo \& Vielliard 1995).

No presente estudo, o método visual responsabilizou-se pela detecção de um maior número de espécies quando comparado aos dois outros métodos utilizados, assinalando $86 \%$ do total das espécies registradas (versus $64 \%$ para o auditivo e $42 \%$ para captura em rede). Possivelmente, esse resultado está relacionado com a composição da avifauna da área estudada, representada predominantemente por espécies de paisagens abertas, muitas delas facilmente registradas por contato visual e outras que mais dificilmente são detectadas através de manifestações sonoras. Sendo assim, esse estudo demonstra que, em levantamentos prolongados e abordando ambientes diferentes, o registro visual, em termos numéricos, suplanta o auditivo, conforme também verificado por Simon (2000) em uma reserva de Mata Atlântica bem conservada na região de Santa Teresa, ES, onde, das 248 espécies registradas, 228 foram detectadas pelo contato visual, 49 das quais unicamente por este método (contra 196/17 pelo método auditivo). Isso ressalta a importância do conhecimento de caracteres morfológicos diagnósticos para evitar os comuns equívocos de identificação

Revista Brasileira de Zoologia 24 (1): 121-132, março 2007 
em inventários de avifauna neotropical, como bem apontado por Willis (2003). Com efeito, a captura e/ou coleta de espécimes visando ao exame minucioso de seus detalhes morfológicos deveriam ser entendidas como uma atividade imprescindível para a identificação de espécies crípticas, com recomendação inclusive para a deposição de material testemunho em instituições científicas para futura averiguação de sua determinação taxonômica (Foster \& CANnel 1990, Martins 1994, Remsen 1995, Gaunt \& Oring 1997, Vuilleumier 1998, 2000, Rojas-Soto et al. 2002, para uma ampla revisão).

Conforme outros estudos (WhitMan et al. 1997, Borges \& Guilherme 2000), o método por redes foi o que amostrou menos espécies. Suas limitações em relação aos outros métodos já foram discutidas por KARR (1981) e REMSEN \& GoOD (1996), os quais mencionam como uma de suas desvantagens a amostragem basicamente de espécies de subbosque, onde as redes normalmente são montadas, o que pode explicar o resultado obtido no presente estudo ( $42 \%$ da avifauna levantada). Observase, porém, sua contribuição ao conhecimento da composição da avifauna na área de estudo, pela inclusão de espécies que não foram detectadas por nenhum dos outros métodos básicos utilizados. Logo, torna-se desejável a combinação de vários métodos de levantamentos em estudos de caracterização de faunas regionais, como já apontado por outros diferentes trabalhos (Rappole et al. 1998, Bernard 2001, Lajmanovich \& Peltzer 2001, Delevey 2003).

\section{AGRADECIMENTOS}

A Edson Valpassos, diretor do PEFG, que incentivou e autorizou a realização desse estudo. A Stanley N. Lima, Joadir Ganda e Luciano C. Sales, pela ajuda nos trabalhos de campo. A Paulo de Marco Júnior, Andressa Gatti e Francisco Barreto, pelo auxílio na análise dos dados nos programas EstimateS e Statistica. A Sergio Pacheco, que apresentou importantes sugestões ao manuscrito. A André Alves, Eliete R. Bortoloni e Alexandre N. Theodoro (FAESA), pelo apoio logístico. A Marcos Raposo, Jorge Nacinovic e Marlene Hoffmann, pela permissão e auxílio nas consultas às coleções ornitológicas do MNRJ e MBML. A Albert D. Ditchfield e Yuri Leite pela revisão do Abstract. Apoio financeiro: Fundo de Apoio à Ciência e Tecnologia do Município de Vitória (Processo FACITEC No 532.7902/2002). O IBAMA forneceu a permissão de coleta de material zoológico no Estado do Espírito Santo (Licença 099/2003-CGFAU/LIC; Processo 02001.002872/99-13).

\section{REFERÊNCIAS}

Aleixo, A. 2001. Conservação da avifauna da Floresta Atlântica: efeitos da fragmentação e importância de florestas secundárias, p. 199-206. In: J.L.B. AlbuQuerque; J.F. CÂndido JR; F.C. Straube \& A.L. Roos (Eds). Ornitologia e conservação: da ciência às estratégias. Tubarão, Editora Unisul, 341p. Aleixo, A. \& J.M.E. Vielliard. 1995. Composição e dinâmica da avifauna da mata de Santa Genebra, Campinas, São Paulo. Revista Brasileira de Zoologia 12 (3): 493-511.

Azevedo, C.O.; R. Kahada; M.T. Tavares \& N.W. Perioto. 2002. Perfil da fauna de himenópteros parasitóides (Insecta, Hymenoptera) em uma área de Mata Atlântica do Parque Estadual da Fonte Grande, Vitória, ES, Brasil. Revista Brasileira de Entomologia 46 (2): 133-137.

Bernard, E. 2001. Species list of bats (Mammalia, Chiroptera) of Santarém area, Pará State, Brazil. Revista Brasileira de Zoologia 18 (2): 455-463.

Bierregaard, R.O. Jr; T.E. Lovejoy; A.A. Santos \& R.W. Hutchings. 1992. The biological dynamics of tropical rainforest fragments. Bioscience 42 (11): 859-866.

BLAKE, J.G. 1992. Temporal variation in point counts of birds in a lowland wet forest in Costa Rica. Condor 94: 265-275.

Borges, S.H. \& E. Guilherme. 2000. Comunidades de aves em um fragmento florestal urbano em Manaus, Amazonas, Brasil. Ararajuba 8 (1): 17-23.

Chiarello, A.G. 2000. Influência da caça ilegal sobre mamíferos e aves das matas de tabuleiro do norte do estado do Espírito Santo. Boletim do Museu de Biologia Mello Leitão, Nova Série 11/12: 229-247.

Christiansen, M.B. \& E. Pitter. 1997. Species loss in a forest bird community near Lagoa Santa in Southeastern Brazil. Biological Conservation 80 (1): 23-32.

Collar, N.J.; L.P. Gonzaga; N. Krabbe; A. Madroño Nieto; L.G. Naranjo; T.A. Parker III \& D.C. Wege. 1992. Threatened Birds of the Americas: the ICBP/IUCN Red Data Book. Cambrigde, International Council for Bird Preservation, 1150 .

ColwelL, R.K. 1997. EstimateS: Statistical estimation of species richness and shared species from samples. Available in the World Wide Web at: http://viceroy.eeb.uconn.edu/ estimates. [Accessed in 26.VIII.2004]

Comitê Brasileiro de Registros Ornitológicos. 2005. Listas das aves do Brasil. Versão 1/2/2005. Disponível na World Wide Web em: http://www.ib.usp.br/cbro. [Acessado em 02.II. 2005]

Conservation International do Brasil; Fundação SOS Mata Atlântica; Fundação Biodiversitas; Instituto de Pesquisas Ecológicas; Secretaria do Meio Ambiente do Estado de São Paulo \& Instituto Estadual de Florestas-MG. 2000. Avaliação e Ações Prioritárias para a Conservação da Biodiversidade da Mata Atlântica e Campos Sulinos. Brasília, MMA/SBF, 40p.

D’Angelo Neto, S.; N. Venturin; A. Oliveira Filho \& F.A. Costa. 1998. Avifauna de quatro fisionomias florestais de pequeno tamanho (5-8 ha) no Campus da UFLA. Revista Brasileira de Biologia 58 (3): 463-472.

Develey, P.F. 2003. Métodos para estudos com aves, p. 153-168. In: Cullen, L. Jr; Rudran, R. \& Valladares-Padua, C. (Eds). Métodos de estudos em biologia da conservação e manejo da vida silvestre. Curitiba, Editora da Universidade Federal do Paraná, Fundação O Boticário de Proteção à Natu- 
reza, 665p.

Foster, M.S. \& P. F. Cannel. 1990. Bird specimens and documentation: critical data for a critical resource. Condor 92 (2): 277-283.

Fundação SOS Mata Atlântica; Instituto Nacional de Pesquisas Espaciais \& Instituto Sócioambiental. 1998. Atlas da Evolução dos Remanescentes Florestais e Ecossistemas Associados no Domínio da Mata Atlântica no Período 19901995. São Paulo, Fundação SOS Mata Atlântica, Instituto Nacional de Pesquisas Espaciais, Instituto Sócioambiental, 29p.

GAUNT, A.S. \& L. ORING. 1997. Guidelines to the use of wild birds in research. Washington, The Ornithological Council, $134 \mathrm{p}$

Grantsau, R. 1988. Os Beija-flores do Brasil: uma chave de identificação para todas as formas de beija-flores do Brasil. Rio de Janeiro, Expressão e Cultura, 233p.

Iвама. 2003. Nova Lista da Fauna Brasileira Ameaçada de Extinção. Disponível na World Wide Web em: http:// www.mma.gov.br/port/sbf/fauna/index.cfm. [Acessado em 15.I.2004]

Ibge. 1987. Projeto RADAM. V. 34. Folha SE 24 Rio Doce. Rio de Janeiro, 540p.

IPEMA. 2005. Conservação da Mata Atlântica no Estado do Espírito Santo: Cobertura Florestal e Unidades de Conservação. Vitória, IPEMA, 142p.

Johnson, R.R.; B.T. Brown; L.T. Haight \& J.M. Simpson. 1981. Playback recordings as a special avian censusing technique. Studies in Avian Biology 6: 68-75.

KARR, J.R. 1981. Surveying birds with mist nest. Studies in Avian Biology 6: 62-67.

Krebs, C.J. 1999. Ecological Methodology. California, Addison Wesley Longman, Benjamin/Cummings, 620p.

Lajmanovich, R.C. \& P.M. Peltzer. 2001. Evaluación de la diversidad de anfibios de un remanente forestal del valle aluvial del río Paraná (entre ríos-Argentina). Boletín de la Asociación Herpetologica Española 12 (1): 12-17.

Marion, W.R.; T.E. O'Meara \& D.S. Maehr. 1981. Use of playback recordings in sampling elusive or secretive birds. Studies in Avian Biology 6: 81-85.

Marsden, S.J.; M. Whiffin; L. Sadgrove \& P. Guimarães Jr. 2000. Parrot populations and habitat use in and around two lowland Atlantic forest reserves, Brazil. Biological Conservation 96: 209-217.

Martins, U.R. 1994. A Coleção Taxonômica, p. 19-43. In: N. Papavero (Ed.). Fundamentos Práticos de Taxonomia Zoológica. São Paulo, UNESP, 285p.

Meyer de Schauensee, R. 1970. A Guide to the Birds of South America. Philadelphia, Academy of Natural Scienses of Ed. Livingston, XIV+470p.

Murcia, C. 1995. Edge effects in fragmented populations: Implications for conservation. Trends in Ecology and Evolution 10: 28-62.
Parker III, T.A. 1991. On the use of tape records in avifaunal surveys. Auk 108: 443-444.

PARKer III, T.A. \& J.M. Goerck. 1997. The importance of national parks and biological reserves to birds conservation in the Atlantic forest region of Brazil, p. 527-541. In: J.V. REMSEN JR (Ed). Studies in neotropical ornithology honoring Ted Parker. Washington, The American Ornitologistis' Union, Ornithological Monographs 48, 742p.

Parker III, T.A.; D.F. Stotz \& J.F. FitzPatrick. 1996. Ecological and distributational data bases, p. 113-436. In: D.F. Sтотz; J.W. Fitzpatrick, T. A. Parker III \& D.K. Moskovitz (Eds). Neotropical birds: ecology and conservation. Chicago, University of Chicago Press, 478p.

Primack, R.B. \& E. Rodrigues. 2001. Biologia da conservação. Londrina, Ed. Vida, VII+327p.

Rappole, J.H.; K. Winker \& G.V.N. Powell. 1998. Migratory bird habitat use in southern Mexico: Mist nets versus point counts. Journal Field Ornithology 69 (4): 635-643.

REDFORD, K.H. 1992. The empty forest. Biosciense 42: 412-422. REMSEN JR, J.V. 1995.The importance of continued collecting of bird specimens to ornithology and bird conservation. Bird Conservation International 5: 145-180.

Remsen Jr, J.V. \& D.A. Good. 1996. Misuse of data from mist-net captures to assess relative abundance in bird populations. Auk 113 (2): 381-398.

Ribon, R.; J.E. Simon \& G.T. Mattos. 2003. Bird extinctions in Atlantic Forest fragments of the Viçosa Region, Southeastern Brazil. Conservation Biology 17 (6): 1827-1839.

Ridgely, R.S. \& G. Tudor. 1989. The birds of South America 1. Austin, University of Texas Press, XVI+516p.

Ridgely, R.S. \& G. Tudor. 1994. The birds of South America 2. Austin, University of Texas Press, XII+814p.

Rojas-Soto, O.R.; S.L. Aquino; L.A. SÁnchez-Gonzáles \& B.E. Hernández-Baños. 2002. La colecta científica en el Trópico: el caso de las aves de México. Ornitologia Neotropical 13: 209-214.

Saint-Hilaire, A. 1974. Viagem ao Espírito Santo e Rio Doce. Belo Horizonte, Ed. Itatiaia, 121p.

Santos, A.J. 2003. Estimativa de riqueza em espécies, p. 19-41. In: L. Cullen Jr, R. Rudran \& C. Valladares-Padua (org.). Métodos de estudos em Biologia da Conservação e manejo da vida silvestre. Curitiba, Editora da Universidade Federal do PAraná, 665p.

Secretaria Municipal do Meio Ambiente; Instituto de Defesa Agropecuária e Florestal \& CePemar-Engenharia e Meio AmBIENTE. 1996. Plano de Manejo: Parque Estadual da Fonte Grande. Vitória, SEAG-IDAF, SEMMAM, CEPEMAR, 66p.

SICK, H. 1997. Ornitologia brasileira. Rio de Janeiro, Ed. Nova Fronteira, 862p.

Simon, J.E. 2000. Composição da avifauna da Estação Biológica de Santa Lúcia, Santa Teresa-ES. Boletim do Museu de Biologia Mello Leitão, Nova Série 11/12: 149-170.

Simon, J.E. \& S.R. Lima. 2004. Primeiro registro documentado 
de Anhima cornuta (Linnaeus, 1766) (Anseriformes Anhimidae) para o Estado do Espírito Santo, Brasil. Ararajuba 12 (2): 63-64.

Simon, J.E.; S.R. Lima; T.D. Novaes \& A. Alves. 2005. Primeiro registro de Podicephorus major (Boddaert, 1783) (Aves: Podicipedidae) para o Estado do Espírito Santo, Brasil. Boletim do Museu de Biologia Mello Leitão, Nova série 18: 59-63.

Smith, E.P. \& G. Belle. 1984. Nonparametric estimation of species richness. Biometrics 40: 119-129.

SkIRVIN, A.A. 1981. Effect of time of day and time of season on the number of observation and density estimates of breeding birds. Studies in Avian Biology 6: 271-274.

SouzA, D. 2002. All the birds of Brazil: an identification guide. Salvador, Ed. Dall, 356p.

Sтотz, D.F. 1993. Geographic variation in species composition of mixed species flocks in lowland humid forest in Brazil. Papéis Avulsos de Zoologia 38 (4): 61-75.

Straube, F.C. \& A. Urben-Filho. 2001. Análise do conhecimento ornitológico da região noroeste do Paraná e áreas adjacentes, p. 223-229. In: J.L.B. Albuquerque; J.F. CÂndido Jr; F.C. Straube \& A.L. Roos (Eds). Ornitologia e conservação: da ciência às estratégias. Tubarão, Editora Unisul, 341p.

Straube, F.C. \& G.V. Bianconi. 2002. Sobre a grandeza e a unidade utilizada para estimar esforço de captura com utilização de redes-de-neblina. Chiroptera Neotropical 8 (1-2): 150-152.

Venturini, A.C.; A.M.S. Ofranti; J.B.M. Varejão \& P.R. Paz. 1996. Aves e mamíferos da restinga: Parque Estadual Paulo
César Vinha, Setiba, Guarapari, ES. Vitória, Secretaria de Estado de Desenvolvimento Sustentável, 68p.

Vielliard, J.M. 1995a. Canto de aves do Brasil. Campinas, Sociedade Brasileira de Ornitologia, CD.

Vielliard, J.M. 1995b. Guia sonoro das aves do Brasil. Campinas, Sociedade Brasileira de Ornitologia, Fundação O Boticário de Proteção à Natureza, CD 1.

Vuilleumier, F. 1998. The need to collect birds in the Neotropics. Ornitologia Neotropical 9: 269-274.

Vuilleumier, F. 2000. Response: further collecting of birds in the Neotropics is still needed. Ornitologia Neotropical 11: 201-203.

Whitman, A.A.; J.M. Hagan \& N.V.L. BrokaW. 1997. Effects of selection logging on birds in northern Belize. Biotropica 30 (3): 449-457.

Wied-Neuwied, M. 1958. Viagem ao Brasil. São Paulo, Companhia Editora Nacional, XIX+536p.

WiLLIS, E.O. 1979. The composition of avian communities in remanescent woodlots in southern Brazil. Papéis Avulsos de Zoologia 33: 1-25.

WilLIs, E.O. 2003. Bird records in the southern neotropics: on the need to critically check specimens, literature citations and field observations. Ornitologia Neotropical 14: 549552.

Willis, E.O. \& Y. ONiki. 2002. Birds of Santa Teresa, ES, Brazil: Do humans add or subtract species? Papéis Avulsos de Zoologia 42: 193-264.

Zar, J. H. 1999. Biostatistical analysis. New Jersey, Prentice Hall, XII+663p.

Recebido em 09.VI.2006; aceito em 28.II.2007. 